Research Article

\title{
Identification of Two DNMT3A Mutations Compromising Protein Stability and Methylation Capacity in Acute Myeloid Leukemia
}

\author{
Samantha Bruno, ${ }^{1}$ Maria Teresa Bochicchio, ${ }^{2}$ Eugenia Franchini, ${ }^{2}$ Antonella Padella, ${ }^{1}$ \\ Giovanni Marconi, ${ }^{1}$ Andrea Ghelli Luserna di Rorà, ${ }^{2}$ Claudia Venturi, ${ }^{1}$ Maddalena Raffini, ${ }^{1}$ \\ Giovanna Prisinzano, ${ }^{2}$ Anna Ferrari, ${ }^{2}$ Lorenza Bandini, ${ }^{1}$ Valentina Robustelli, ${ }^{1}$ \\ Martina Pazzaglia, ${ }^{1}$ Maria Chiara Fontana, ${ }^{1}$ Chiara Sartor, ${ }^{1}$ Maria Chiara Abbenante, ${ }^{1,3}$ \\ Cristina Papayannidis, ${ }^{1}$ Simona Soverini, ${ }^{1}$ Emanuela Ottaviani, ${ }^{1}$ Giorgia Simonetti ${ }^{D},{ }^{2}$ \\ and Giovanni Martinelli ${ }^{2}$
}

\footnotetext{
${ }^{1}$ Department of Experimental, Diagnostic and Specialty Medicine, University of Bologna, Institute of Hematology "L. e A. Seràgnoli", Bologna, Italy

${ }^{2}$ Istituto Scientifico Romagnolo per lo Studio e la Cura dei Tumori (IRST) IRCCS, Meldola (FC), Italy

${ }^{3}$ Casa Sollievo della Sofferenza, San Giovanni Rotondo, Italy
}

Correspondence should be addressed to Giorgia Simonetti; giorgia.simonetti@irst.emr.it

Received 26 April 2019; Accepted 11 September 2019; Published 30 October 2019

Academic Editor: San-Lin You

Copyright () 2019 Samantha Bruno et al. This is an open access article distributed under the Creative Commons Attribution License, which permits unrestricted use, distribution, and reproduction in any medium, provided the original work is properly cited.

\begin{abstract}
Somatic mutations of DNMT3A occur in about $20 \%$ of acute myeloid leukemia (AML) patients. They mostly consist in heterozygous missense mutations targeting a hotspot site at R882 codon, which exhibit a dominant negative effect and are associated with high myeloblast count, advanced age, and poor prognosis. Other types of mutations such as truncations, insertions, or singlenucleotide deletion also affect the DNMT3A gene, though with lower frequency. The present study aimed to characterize two DNMT3A gene mutations identified by next-generation sequencing (NGS), through analysis of protein stability and DNA methylation status at $\mathrm{CpG}$ islands. The first mutation was a single-nucleotide variant of DNMT3A at exon 20 causing a premature STOP codon (c.2385G > A; p.Trp795*; NM_022552.4). The DNMT3A mutation load increased from $4.5 \%$ to $38.2 \%$ during guadecitabine treatment, with a dominant negative effect on $\mathrm{CpG}$ methylation and on protein expression. The second mutation was a novel insertion of 35 nucleotides in exon 22 of DNMT3A (NM_022552.4) that introduced a STOP codon too, after the amino acid Glu863 caused by a frameshift insertion (c.2586_2587insTCATGAATGAGAAAGAGGACATCTTATGGTGCACT; p. Thr862_Glu863fsins). The mutation, which was associated with reduced DNMT3A expression and CpG methylation, persisted at relapse with minor changes in the methylation profile and at protein level. Our data highlight the need to better understand the consequences of DNMT3A mutations other than R882 substitutions in the leukemogenic process in order to tailor patient treatments, thus avoiding therapeutic resistance and disease relapse.
\end{abstract}

\section{Introduction}

Genetic alterations of chromatin regulators and epigenetic modifications cooperate to the pathogenesis of acute myeloid leukemia (AML) [1]. Among epigenetic modifications, DNA methylation represents a mechanism adopted by cells to regulate DNA accessibility through the conversion of 5methylcytosine (5-mC) to 5-hydroxymethylcytosine (5$\mathrm{hmC}$ ). It switches on/off the promoter of several target genes, thus regulating their expression levels and contributing to disease phenotype. DNA methyltransferases (DNMT3A, DNMT3B, DNMT1, and DNMT3L) are the key 
enzymes involved in DNA methylation. DNMT3A and DNMT3B produce de novo hemimethylated DNA and de novo symmetric DNA methylation, respectively, while DNMT1 is involved in maintenance of DNA methylated status $[2,3]$.

DNA methylation has been identified as a specific functional category of mutated genes in AML that includes mutation in DNMT3A, DNMT3B, DNMT1, Tet methylcytosine dioxygenase 1 and 2 (TET1, TET2), and isocitrate dehydrogenase 1 and 2 (IDH1, IDH2) genes [4]. Moreover, Wilms tumor 1 (WT1) mutations were shown to reduce DNA methylation levels significantly in AML patients, conferring the same hypomethylation signature observed in patients harbouring TET2 mutations [5]. Haploinsufficiency of WT1 in preclinical models was also shown to decrease 5$\mathrm{hmC}$ levels and impair TET2 function, especially in elderly animals [6].

DNMT3A mutations are among the most frequent driver mutations in AML, third for frequencies to Fms-like tyrosine kinase 3 (FLT3) and nucleophosmin 1 (NPM1) [1]. About $20 \%$ of patients with de novo AML show recurrent somatic mutations in the DNMT3A gene [7], which represents an early lesion in leukemia development. Indeed, DNMT3A genomic alterations are considered a preleukemic event in hematopoietic stem cells (HSCs) and confer a proliferation advantage [8]. They have been identified in preleukemic stem cells of myelodysplastic syndrome (MDS) and AML patients [9]. Moreover, mutations in genes encoding epigenetic modifiers, including TET2, ASXL transcriptional regulator 1 (ASXL1), and DNMT3A have been reported in aging healthy people and are considered the first "hit" for the "clonal hematopoiesis of indeterminate potential (CHIP)" $[10,11]$.

Genomic lesions of the DNMT3A gene mostly consist in missense mutations targeting a hotspot site at R882 codon, which exhibits a dominant negative effect [12]. Furthermore, DNMT3A R882 mutations are associated with a hypomethylated status of $\mathrm{CpG}$ islands, higher relapse rate, and poor prognosis [13-18], and they persist during remission $[19,20]$. It has been recently shown that the methylation levels are dependent on the amount of active DNMT3A and higher methylation confers a better prognosis to AML patients [21]. Among patients carrying DNMT3A mutations, $15-20 \%$ do not show the substitution at R882 codon, but they harbour truncations, insertion, or single-copy deletions $[1,7]$.

The present study aimed to characterize two undescribed DNMT3A mutations identified by next-generation sequencing (NGS) in two different AML patients, through analysis of protein stability and DNA methylation status at $\mathrm{CpG}$ islands.

\section{Materials and Methods}

2.1. Patients. Samples were obtained from AML patients after informed consent was approved by the Institutional Ethical Committee (protocol 112/2014/U/Tess of Policlinico Sant'Orsola-Malpighi) in accordance with the Declaration of Helsinki.
2.2. Isolation of Mononuclear Cells. Bone marrow (BM) mononuclear cells were isolated by Ficoll density-gradient (Amersham Biosciences) and lysed in guanidine-thiocyanate-containing lysis buffer (RLT, Qiagen, Ltd).

2.3. Genomic DNA, RNA, and Protein Extraction. The AllPrep DNA/RNA/Protein Mini Kit (Qiagen, Ltd) was used to extract DNA, RNA, and protein from primary mononuclear cells according to the manufacturer's instructions.

\subsection{Ion Torrent Next-Generation Sequencing and Variant} Calling. The patients' mutational profile was determined using Oncomine Myeloid Research Assay. The libraries were quantified using the Ion Library TaqMan TM Quantitation Kit (Thermo Fisher Scientific) following manufacturer's instructions and run in the Ion $530^{\mathrm{TM}}$ chip on the Ion Torrent S5 instrument (Thermo Fisher Scientific). Sequence alignment and analysis were performed using the Ion Torrent Suite Software v.5.8.0 and the Ion Reporter software v.5.10.3.0 and v.5.10.5.0 (Thermo Fisher Scientific). Human genome build 19 (Hg 19) was used as the reference for sequence alignment. A minimum coverage depth per amplicon of 500 was required; nonsynonymous mutations with a variant allele frequency $(\mathrm{VAF}) \geq 2.5 \%$ were reported; $5^{\prime}$ and $3^{\prime}$ untranslated regions (UTRs), intronic donor splice-site variants, and polymorphisms were filtered out.

\subsection{Amplification and Sanger Sequencing. DNMT3A mu-} tations were confirmed by Sanger sequencing. Briefly, reverse transcription was performed starting from $1 \mu \mathrm{g}$ of RNA using reverse transcriptase M-MuLV enzyme (SigmaAldrich $\left.{ }^{\circledR}\right)$. The obtained cDNA was used to perform polymerase chain reaction (PCR) using Applied Biosystem AmpliTaq Gold ${ }^{\circledR}$ (Thermo Fisher Scientific) and the following primers: $\mathrm{FW}: 5^{\prime}$-TCGAGTCCAACCCTGTGATG-3' and REV: 5'-TAACTTTGTGTCGCTACCT CAGTT- $3^{\prime}$. Cycling conditions were as follows: 10 minutes at $95^{\circ} \mathrm{C} ; 40$ cycles: 30 seconds at $94^{\circ} \mathrm{C} ; 45$ seconds at $54^{\circ} \mathrm{C} ; 30$ seconds at $72^{\circ} \mathrm{C}$; and 10 minutes at $72^{\circ} \mathrm{C}$. PCR amplicons were purified using Applied Biosystems ExoSAP-IT ${ }^{\mathrm{TM}}$ PCR Product Cleanup Reagent (Thermo Fisher Scientific) according to manufacturer's instructions. Samples were sequenced according to dideoxy procedure BigDye ${ }^{\circledR}$ Terminator v3.1 Cycle Sequencing Kit (Thermo Fisher Scientific) on an Applied Biosystems 3730xL genetic analyzer system (Thermo Fisher Scientific).

2.6. Capillary Electrophoresis. The allelic ratio of FLT3-ITD was measured by capillary electrophoresis. The reaction was performed starting from $20 \mu \mathrm{g}$ of DNA, by using AmpliTaq ${ }^{\mathrm{TM}}$ Gold DNA polymerase enzyme, Buffer II, magnesium chloride (Thermo Fisher Scientific) and the following primers: forward, 5'-GCAATTTADGTATGAAAGCCAGC- ${ }^{\prime}$, and reverse, $5^{\prime}$-CTTTCAGCATTTTGACGGCAACC- $3^{\prime}$. Cycling conditions were 10 minutes at $95^{\circ} \mathrm{C} ; 30$ seconds at $95^{\circ} \mathrm{C} ; 60$ seconds at $60^{\circ} \mathrm{C} ; 60$ seconds at $72^{\circ} \mathrm{C}$ for 35 
cycles; 7 minutes at $72^{\circ} \mathrm{C}$. The amplification products were sequenced on Applied Biosystems 3130 Genetic Analyzer (Thermo Fisher Scientific) and were analyzed with GeneMapper $^{\mathrm{TM}}$ Software 5 (Thermo Fisher Scientific). The ratio of the area of mutated and wildtype (wt)-FLT3 defined the allelic ratio.

2.7. Western Blot Analysis. Protein extracts were separated by sodium dodecyl sulphate-polyacrylamide gel electrophoresis (SDS-PAGE, Bio-Rad) and transferred onto nitrocellulose membranes. The following antibodies were used: rabbit anti-DNMT3A (D23G1; Cell Signaling Technologies) and goat anti- $\beta$-actin (Santa Cruz Biotechnology) as control; horseradish peroxidase (HRP)-conjugated antirabbit immunoglobulin (Ig)G (GE Healthcare) and anti-goat IgG (Santa Cruz) as secondary antibodies. ECL Prime (GE Healthcare) reagent was used for detection using ChemiDoc XRS+ System (Bio-Rad). Signal quantification was performed using Image J software.

2.8. DNA Methylation Assay. Methylation was quantified on total DNA using the MethylFlash Methylated DNA 5-mC Quantification Kit (Epigentek) using triplicates of $100 \mathrm{ng}$ of DNA from each sample. The absorbance (OD) was read at $450 \mathrm{~nm}$ using the Multiskan EX automatic microplate reader (Thermo Fisher Scientific). To quantify the absolute amount of methylated DNA, we generated a standard curve using a negative control (ME3) and 5 dilutions of positive control $(10.0 ; 5.0 ; 2.0 ; 1.0 ; 0.5 \mathrm{ng} / \mu \mathrm{l})$; next we determined the slope $(\mathrm{OD} / \mathrm{ng})$ using linear regression. According to manufacturer's instructions, we used the following formulas to calculate the amount and percentage of 5-mC:

$$
\begin{aligned}
5 \mathrm{mC}(\mathrm{ng}) & =\frac{(\text { sample OD }-\mathrm{ME} 3 \mathrm{OD})}{\text { slope } \times 2^{*}}, \\
5 \mathrm{mC}(\%) & =\frac{5 \mathrm{mC}(\mathrm{ng})}{\text { sample DNA }(\mathrm{ng}) \times 100},
\end{aligned}
$$

Where ${ }^{*} 2$ is a factor to normalize the positive control because it contains only $50 \%$ of $5 \mathrm{mC}$.

\section{Results and Discussion}

3.1. Identification of a Premature Stop Codon in Exon 20 of the DNMT3A Gene in AML. NGS on a primary AML sample identified a single-nucleotide variant of DNMT3A at exon 20 causing a premature STOP codon (c.2385G > A; p.Trp795Ter) that was confirmed by Sanger sequencing (Figure 1(a)). Mutations at codon 795 were previously reported in angioimmunoblastic T-cell lymphoma (p.Trp795_Gly796ins3) [22] and in refractory anemia evolving to secondary AML (Trp795Cys) [23]. A STOP codon at position 795 has been reported in the Leiden Open Variation Database (c.2384G > A, https://www.lovd.nl/) and the c.2385G > A mutation has been found annotated in the database of single-nucleotide polymorphisms (dbSNP, rs1395575712). Moreover, missense variants affecting the codon 795 (chr2:25462024:A > C and chr2:25462024:
$A>G)$ were also annotated in the Genome Aggregation Database (gnomAD) with an allelic frequency of 0.000003979 and 0.000007957 , respectively (https:// gnomad.broadinstitute.org/). We detected the mutation $(\mathrm{VAF}=4.5 \%)$ in the $\mathrm{BM}$ sample of a 74 -year-old woman (AML\#1), with $70 \%$ of AML blasts at diagnosis, normal karyotype, wt-FLT3, NPM1, and tumor protein p53 (TP53) and intermediate cytogenetic risk according to ELN 2017 [24]. Mutations of IDH2 (c.515G > A; p.Arg172Lys), BCL6 corepressor (BCOR) (c.2915_2916insA; p.Tyr972Ter), and TET2 (c.3641G > A; p.Arg1214Gln) were also detected in the patient, with a VAF of $6.1 \%, 4.8 \%$, and $3.2 \%$, respectively (Figure 1(b) and Table 1). Previous evidence reported the co-occurrence of DNMT3A lesions with mutations of NPM1, IDH2, and TP53 and with those affecting genes involved in chromatin and splicing in AML cases [25]. The patient received an induction therapy with the DNMT inhibitor guadecitabine [26], with persistence of disease at the bone marrow evaluation after 4 courses of therapy ( $40 \%$ of blasts and stable cytogenetic risk). The DNMT3A mutation load increased to $38.2 \%$ in the sample evaluated 4 months after treatment. NGS analysis revealed an increasing VAF during treatment of the IDH2 $(6.1 \%$ to $39.8 \%$ ) and BCOR mutations ( $4.8 \%$ to $42.1 \%$ ) detected at diagnosis, along with the emergence of a TP53 mutation (c.607G > A; p.Val203Met, VAF 5.3\%, Figure 1(b) and Table 1), which was not detectable at diagnosis. Nowadays, no predictive marker of response to guadecitabine has been defined for newly diagnosed AML. However, Chung et al. reported no significant association between gene mutations and complete remission in a cohort of 128 relapsed/refractory AML [27]. Trends were observed for TET2-mutated cases (higher CR rate) and IDH1/2-mutated and TP53-mutated AML (resistance). Moreover, TET2 mutations have been associated with increased response to hypomethylating agents in MDS $[28,29]$ and AML with low blast count [28]. We believe that our results suggest the persistence during treatment of a minor clone harbouring DNMT3A, IDH2, and BCOR mutations, which was positively selected and progressively expanded, along with the acquisition of a novel TP53-mutated subclone. In parallel, TET2-mutated blasts were killed by the treatment.

3.2. Detection of a Novel 35 Nucleotides Insertion in Exon 22 of the DNMT3A Gene in AML. Targeted deep sequencing leads to the identification of an additional DNMT3A genetic alteration consisting in a new insertion of 35 nucleotides in exon 22 of the DNMT3A gene causing the amino acid change Glu863Ser followed by a premature STOP codon (c.2586_2587ins35:TC ATGAATGAGAAAGAGGACATCTTATGGTGCAC; p. Thr862_Glu863fsins, Figure 2(a)). The variant, which has never been reported before, was detected in primary leukemic cells isolated from a 63-year-old woman (AML\#2), with $80 \%$ of blasts in the BM at diagnosis and $90 \%$ at relapse. The patient had normal karyotype, low FLT3ITD allelic burden (c.1747_1748ins57: GCTCCTCAGATAATGAGTACTTCTACGTTGATTTCAGAGAATATGAATATGATCCAA; VAF: $11.7 \%$ at diagnosis), and 


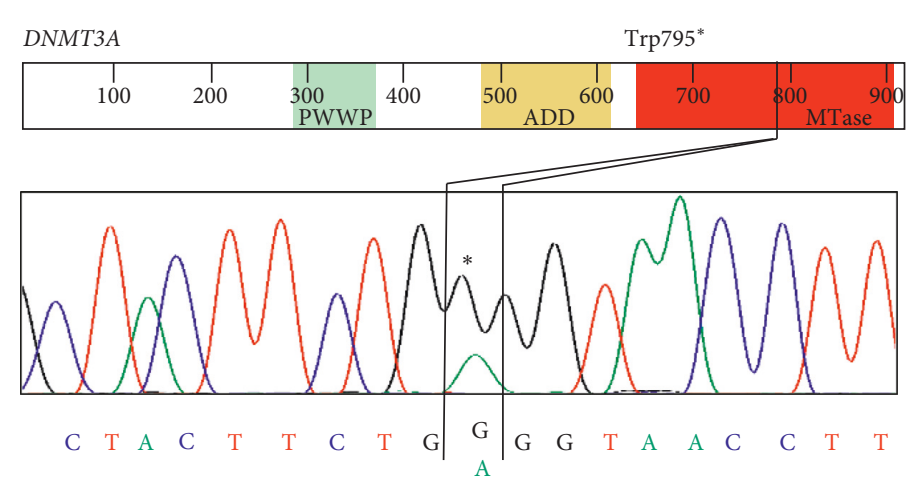

(a)

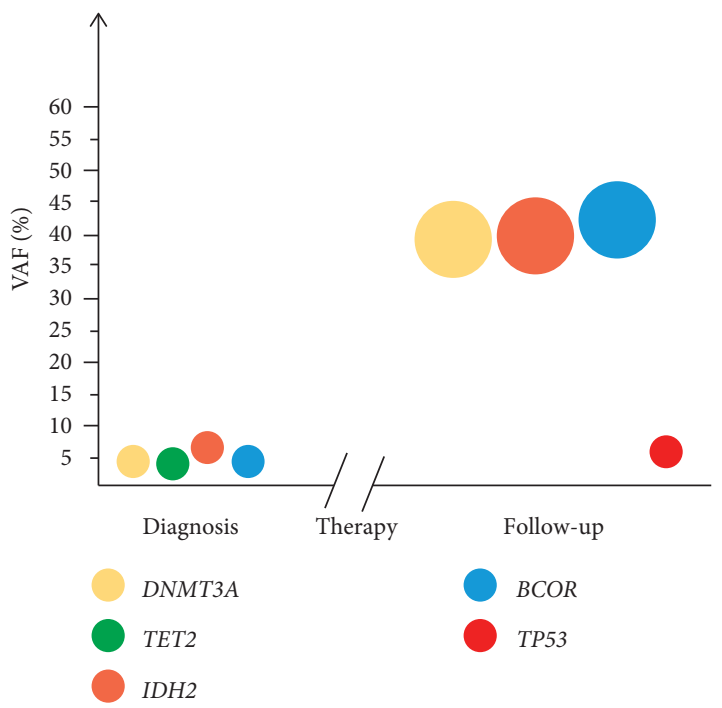

(b)

FIGURE 1: Sanger sequencing of the DNMT3A single-nucleotide variant identified in AML\#1 and analysis of clonal evolution based on NGS data. (a) AML\#1 showed a single-nucleotide variant of DNMT3A at exon 20 causing a premature STOP codon (c.2385G > A; p.Trp795*; NM_022552.4). (b) Variant allele frequency (VAF) changes of the detected mutations at diagnosis and follow-up (after 4 courses of guadecitabine therapy), showing expansion of the DNMT3A-mutated clone.

TABle 1: Mutational profile of AML\#1 at diagnosis and follow-up.

\begin{tabular}{|c|c|c|c|c|c|c|c|c|c|}
\hline $\mathrm{Pt}$ & Gene & Locus & NM_ID & Exon & Type & Coding & $\begin{array}{c}\text { Amino acid } \\
\text { change }\end{array}$ & VAF (\%) & $\begin{array}{c}\text { Variant } \\
\text { effect }\end{array}$ \\
\hline \multirow{4}{*}{ AML\#1Dx } & DNMT3A & chr2:25462022 & NM_022552.4 & 20 & SNV & c. $2385 \mathrm{G}>\mathrm{A}$ & p.Trp795Ter & 4.45 & Nonsense \\
\hline & TET2 & chr4:106164773 & NM_001127208.2 & 6 & SNV & c. $3641 \mathrm{G}>\mathrm{A}$ & p.Arg & 3.20 & ense \\
\hline & IDH2 & chr15:90631837 & NM_002168.3 & 4 & SNV & c. $515 \mathrm{G}>\mathrm{A}$ & p.Arg & 6.06 & ense \\
\hline & BCOR & ChrX:39931683 & NM_001123385.1 & 4 & INDEL & c. $2915 \_2916 \mathrm{ins} A$ & p.Tyr & 4.76 & Nor \\
\hline \multirow{4}{*}{ AML\#1F-UP } & DNMT3A & chr2:25462022 & NM̄_022552.4 & 20 & SNV & c. $2385 \mathrm{G}>\mathrm{A}$ & p. $\operatorname{Trp} 7$ & 38.20 & Nonsense \\
\hline & IDH2 & chr15:90631837 & NM_002168.3 & 4 & SNV & c. $515 \mathrm{G}>\mathrm{A}$ & p.Arg172Lys & 39.80 & Missense \\
\hline & TP53 & chr17:7578242 & NM_000546.5 & 6 & SNV & c. $607 \mathrm{G}>\mathrm{A}$ & p.Val203Met & 5.25 & Missense \\
\hline & $B C O R$ & chrX:39931683 & NM_001123385.1 & 4 & INDEL & c. $2915 \_2916 \mathrm{ins} A$ & p.Tyr972Ter & 42.08 & Nonsense \\
\hline
\end{tabular}

Pt: patient; Dx: diagnosis; F-UP: follow-up; SNV: single-nucleotide variant; INDEL: insertion/deletion; ins: insertion.

DNMT3A p. Thr862_Glu863fins

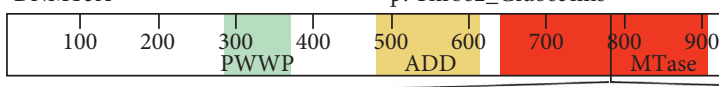

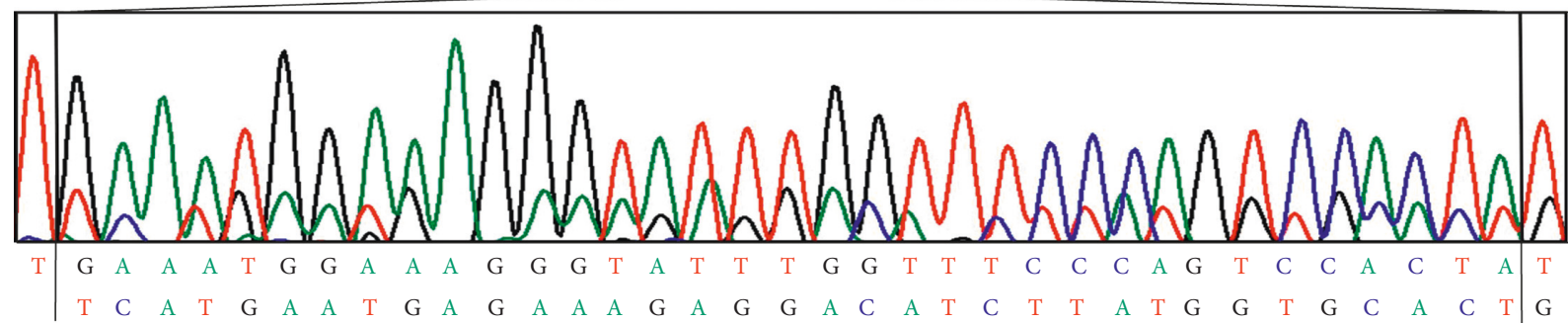

(a)

FIGURE 2: Continued. 


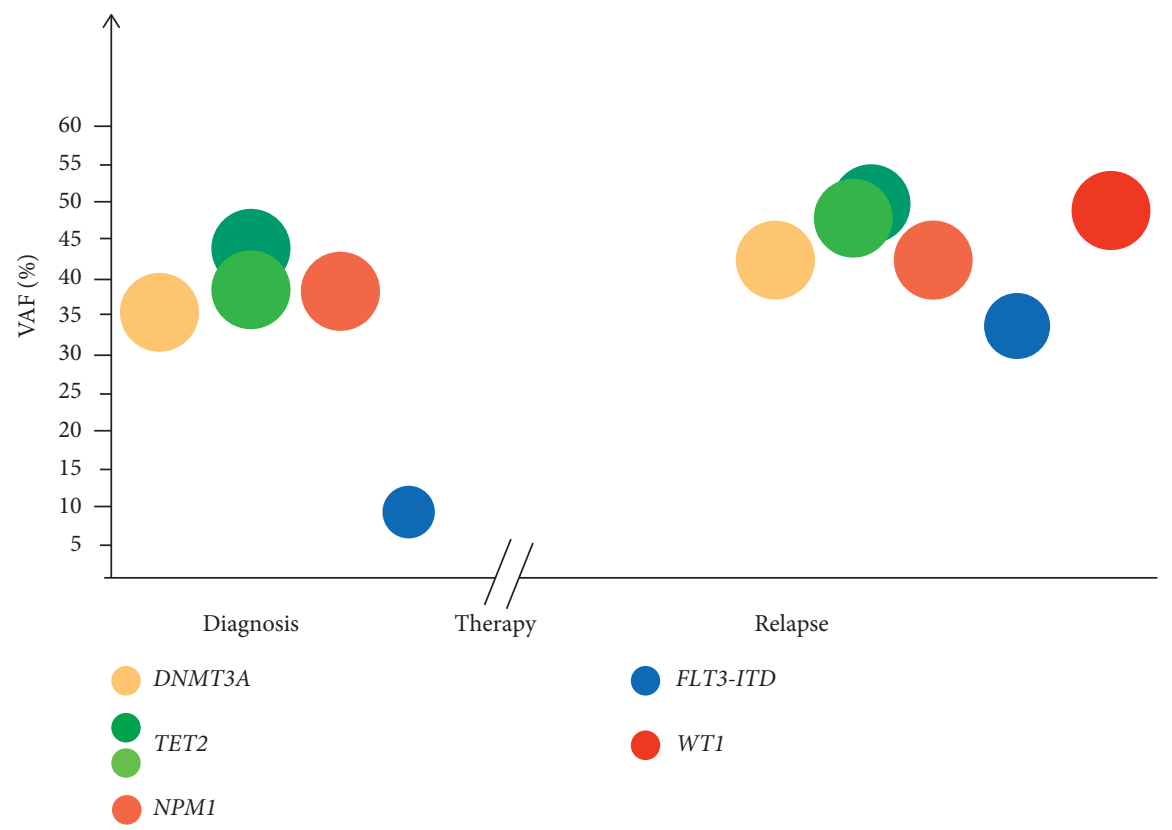

(b)

FIGURE 2: Sanger sequencing of the insertion in the DNMT3A gene identified in AML\#2 and analysis of clonal evolution based on NGS data. (a) AML\#2 showed a novel insertion of 35 nucleotides in the exon 22 of the DNMT3A gene (c.2586_2587ins35: TCATGAATGAG AAAGAGGACATCTTATGGTGCAC; p Thr862_Glu863fsins). (b) Representation of clonal evolution of AML\#2 from diagnosis to relapse (that occurred after 7 months of complete remission achieved with FLAI-5 chemotherapy regimen).

intermediate cytogenetic risk according to the ELN 2017 classification [24]. Molecular analysis also detected mutations of NPM1 (c.863_864insCTTG; p.Trp288fs; VAF 37.4\%) and TET2 (c.395delA; p.Asn132fs; VAF $38.6 \%$ and c.5504delG; p.Gly1835fs; VAF 43.6\%, Figure 2(b) and Table 2). The patient started fludarabine, arabinosyl cytosine, and idarubicin (FLAI-5) induction regimen [30, 31] and obtained complete hematological remission one month later, with undetectable FLT3 mutations. After 7 months of complete remission, the patient relapsed. Mutational analysis performed on the diagnosis and relapse samples showed that the DNMT3A mutation loads were, respectively, $35.8 \%$ and $42.5 \%$. The relapse sample presented an increase of $5.5 \%$ for VAF of NPM1 mutation, along with an expanded FLT3-ITD clone ( $11.7 \%$ to $40.1 \%)$, the persistence of TET2 mutations with a slight VAF increase (c.395delA; p.Asn132fs; VAF $48.4 \%$ and c.5504delG; p.Gly1835fs; VAF 49.7\%), acquisition of a WT1 mutation (c.1109G > C; p.Arg370Pro; VAF: 48.3\%; Figure 2(b) and Table 2). In this patient, the treatment was not able to eradicate the leukemic clone harbouring TET2, DNMT3A, and NPM1 mutations, which seems to have acquired an additional WT1 mutation at relapse. Moreover, AML relapse was characterized by the expansion of the FLT3-ITD clone that was reduced but not eradicated by the treatment, with an increase of allelic ratio from 0.09 (diagnosis) to 0.21 (relapse). A recent study showed that the persistence of $D N M T 3 A$ mutation at a VAF $\geq 2 \%$ at first remission is a common event in AML, occurring in 65\% of cases with mutation at diagnosis and is associated with older age and inferior relapse-free survival [32].
3.3. The Identified DNMT3A Mutations Alter DNA CpG Islands Methylation and Protein Stability. To investigate the functional consequences of the identified mutations, we performed methylation analysis of CpG islands on DNA extracted from primary leukemic samples of both patients. Our cases showed DNA hypomethylation in comparison with primary AML samples with wt-DNMT3A gene or R882H mutation (VAF 37.0\%) (Figure 3(a)). In the sample carrying Trp795* mutation, CpG islands methylation shifted from $47.1 \%$ at diagnosis to $24.4 \%$ at follow-up $(p=0.020)$, in accordance with the increase in the mutations allelic burden (from $4.5 \%$ at diagnosis to $38.2 \%$ at follow-up). At follow-up, CpG methylathion was significantly reduced compared with the wt-DNMT3A sample $(p=0.017)$. However, the observed hypomethylation status may be associated both with hypomethylating agent treatment and with the expansion of the DNMT3A-mutated clone during treatment.

In the sample carrying the insertion at Thr862_Glu863, CpG island methylation at diagnosis was significantly reduced compared with the wt-DNMT3A sample ( $p=0.047)$. Moreover, its levels were similar to those observed in the $\mathrm{R} 882 \mathrm{H}$ sample. No major changes occurred between disease diagnosis and relapse in terms of $\mathrm{CpG}$ methylation level and DNMT3A (c.2586_2587ins35; p. Thr862_Glu863fsins) VAF. The results suggest that the identified mutations induce DNMT3A loss of function, similarly to the $\mathrm{R} 882 \mathrm{H}$ alteration. It has been recently shown that hypomethylation is an initiating feature of AML with $D N M T 3 A^{\mathrm{R} 882}$ [33] and that demethylator phenotypes, which are partially related to DNMT3A mutational status, have a prognostic role, independent of age and cytogenetic abnormalities [34]. Future 
TABLE 2: Mutational profile of AML\#2 at diagnosis and relapse.

\begin{tabular}{|c|c|c|c|c|c|c|c|c|c|}
\hline $\mathrm{Pt}$ & Gene & Locus & NM_ID & Exon & Type & Coding & $\begin{array}{l}\text { Amino acid } \\
\text { change }\end{array}$ & $\begin{array}{l}\text { VAF } \\
(\%)\end{array}$ & $\begin{array}{l}\text { Variant } \\
\text { effect }\end{array}$ \\
\hline \multirow{5}{*}{ AML\#2Dx } & DNMT3A & $\begin{array}{c}\text { chr2: } \\
25458586\end{array}$ & NM_022552.4 & 22 & INDEL & c.2586_2587ins* & p.Glu863Ser & 35.79 & Nonsense \\
\hline & TET2 & $\begin{array}{c}\text { chr4: } \\
106155491\end{array}$ & NM_001127208.2 & 3 & INDEL & c.395delA & p.Asn132fs & 38.55 & fs del \\
\hline & TET2 & $\begin{array}{c}\text { chr4: } \\
106197168\end{array}$ & NM_001127208.2 & 11 & INDEL & c.5504delG & p.Gly1835fs & 43.60 & fs del \\
\hline & NPM1 & $\begin{array}{c}\text { chr5: } \\
170837545\end{array}$ & NM_002520.6 & 11 & INDEL & c.863_864insCTTG & p.Trp288fs & 37.41 & fs ins \\
\hline & FLT3 & $\begin{array}{c}\text { chr13: } \\
28608308\end{array}$ & NM_004119.2 & 14 & INDEL & c.1747_1748ins ${ }^{* *}$ & p.Gly583_Ser584ins s** $^{* *}$ & 11.70 & Nonfs ins \\
\hline \multirow{6}{*}{$\mathrm{AML} \# 2 \mathrm{R}$} & DNMT3A & $\begin{array}{c}\text { chr2: } \\
25458586\end{array}$ & NM_022552.4 & 22 & INDEL & c.2586_2587ins* & p.Glu863Ser & 42.45 & Nonsense \\
\hline & TET2 & $\begin{array}{c}\text { chr4: } \\
106155491\end{array}$ & NM_001127208.2 & 3 & INDEL & c.395delA & p.Asn132fs & 48.34 & fs del \\
\hline & TET2 & $\begin{array}{c}\text { chr4: } \\
106197168\end{array}$ & NM_001127208.2 & 11 & INDEL & c.5504delG & p.Gly1835fs & 49.65 & fs del \\
\hline & NPM1 & $\begin{array}{c}\text { chr5: } \\
170837545\end{array}$ & NM_002520.6 & 11 & INDEL & c.863_864insCTTG & p.Trp288fs & 42.87 & fs ins \\
\hline & WT1 & $\begin{array}{c}\text { chr11: } \\
32417943\end{array}$ & NM_024426.4 & 7 & SNV & c. $1109 \mathrm{G}>\mathrm{C}$ & p.Arg370Pro & 48.25 & Missense \\
\hline & FLT3 & $\begin{array}{c}\text { chr13: } \\
28608308\end{array}$ & NM_004119.2 & 14 & INDEL & c.1747_1748ins ${ }^{* *}$ & p.Gly583_Ser584ins ${ }^{* * *}$ & 40.10 & Nonfs ins \\
\hline
\end{tabular}

Pt: patient; Dx: diagnosis; R: relapse; SNV: single-nucleotide variant; INDEL: insertion/deletion; ins: insertion; fs: frameshift; del: deletion; *insertion of 35 nucleotides: TCATGAATGAGAAAGAGGACATCTTATGGTGCAC; ${ }^{* *}$ insertion of 57 nucelotides: GCTCCTCAGATAATGAGTACTTCTACGTTGATTTCAGAGAATATGAATATGATCCA; ${ }^{* * *}$ SerSerAspAsnGluTyrPheTyrValAspPheArgGluTyrGluTyrAspProSer.

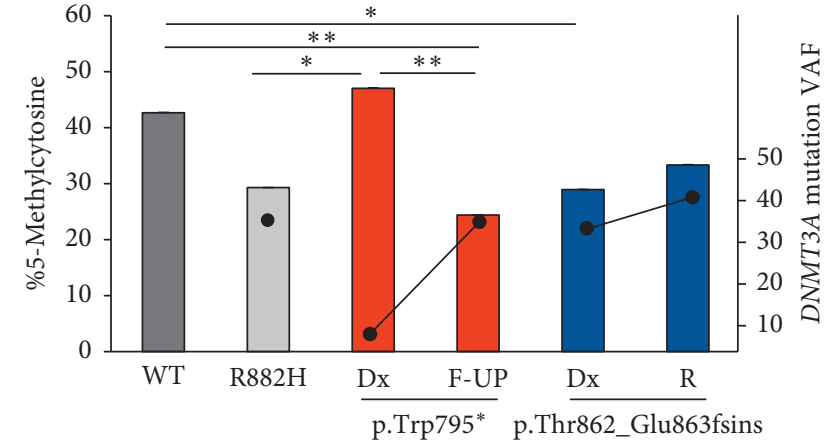

(a)

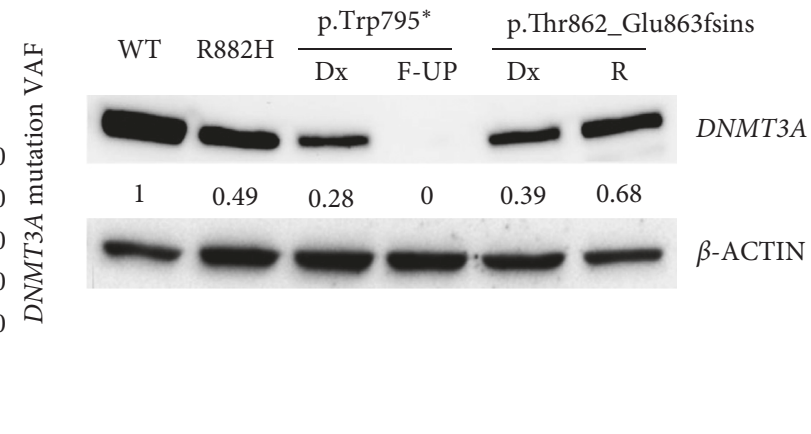

(b)

Figure 3: Effect of DNMT3A mutations on protein function and expression. (a) CpG islands methylation analysis. The bars represent mean \pm standard deviation (SD) of the absolute 5-mC level (percentage of 5-mC left axis; SD values are barely detectable due to low values). Dots represent the DNMT3A VAF in the analyzed samples (right axis). A BM sample from an AML patient harbouring the DNMT3A R882H mutation (VAF 37.0\%) was used for comparison (wt: wildtype; R882H: canonical mutation; Dx: diagnosis; F-UP: follow-up; R: relapse). (b) Western blot analyses of DNMT3A expression in representative AML cases with wt-DNMT3A and R882H mutation, followed by diagnosis and follow-up of AML\#1, diagnosis and relapse of AML\#2 (wt: wildtype; Dx: diagnosis; F-UP: follow-up; R: relapse). $\beta$-actin was used for loading normalization and quantification. Numbers represent DNMT3A levels normalized on the wt sample.

analyses of the aberrant DNA methylation pattern may help define specificities compared with DNMT3A ${ }^{\mathrm{R} 882}$ AML and novel potential silenced or activated enhancers involved in leukemogenesis [35].

To understand whether alterations in DNMT3A protein expression were responsible for the observed changes in CpG methylation, we performed western blot on BM samples at different time points. Protein analysis revealed that despite the low DNMT3A mutation burden in the diagnosis sample of the case AML\#1 (Trp795*), DNMT3A protein levels were reduced to $72 \%$ and $21 \%$ compared with wt-DNMT3A and $\mathrm{R} 882 \mathrm{H}$ samples, respectively (Figure 3(b)). Furthermore, the increased VAF of truncated DNMT3A was associated with no detectable protein in the follow-up sample (Figure 3(b)). Despite the heterozygous mutational status, the wt protein isoform became undetectable. We speculated that the wt protein was unable to form a heterodimer with the truncated DNMT3A form, 
which may also be unstable, thus causing a premature degradation. This hypothesis is supported by a recent study, which demonstrates that DNMT3A truncation mutations have a dominant negative effect with loss of function and haploinsufficiency in AML [12]. On the contrary, DNMT3A protein was detectable in the analyzed samples from AML\#2 (p.Thr862_Glu863fsins). In this patient, we observed that DNMT3A levels were reduced in the diagnosis sample compared with the wt one and were similar to those obtained in the R882H-mutated case (Figure 3(b)). DNMT3A expression slightly increased in the relapse sample, showing a $30 \%$ reduction in protein level compared with the wt sample. The data suggest that the mutation, which is predicted to introduce a premature STOP codon, interferes with protein expression but does not alter the stability of the wt protein. Future investigation will be useful to demonstrate if the presence of a portion of the catalytic domain in this truncated protein allows it to bind to wt-DNMT3A. Taken together, the protein and methylation analyses indicate that this mutation results in decreased DNMT3A expression and function.

\section{Conclusions}

DNMT3A mutations are becoming highly relevant in hematological malignancies, thanks to NGS technologies which allow us to better characterize myeloid disorders. In this study, we presented two mutations of the DNMT3A gene never described in AML and investigated their consequences on protein expression and function. Both mutations localized in the catalytic domain of the DNMT3A protein and were predicted to cause loss of function. The first one is responsible for a truncated, nondetectable protein associated with hypomethylation of $\mathrm{CpG}$ islands, which expanded under the pressure of hypomethylating agent treatment. The second mutation was an insertion of 35 nucleotides, with a hypomethylation pattern, suggesting a negative effect on $\mathrm{CPG}$ methylation mediated by mutant DNMT3A. The DNMT3A-mutated clone escaped therapy selection and likely acquired a WT1 mutation. Both patients showed evidence of clonal evolution, resulting from the selective pressure induced by treatment. Our strategy, based on bulk sequencing, allows us to draw a picture of AMLrelated mutations, along with their frequency and to speculate on mutation co-occurrence at disease diagnosis and progression. However, a single-cell sequencing approach is needed to precisely evaluate the clonal complexity of AML across the different disease stages, drive conclusions on the clonal and subclonal architecture, and uncover genomic trajectories of leukemia evolution [36-39]. The presence of cooperating mutations and their contribution to the leukemic phenotype along with the identified DNMT3A mutations deserve further investigation. Our data highlight the need to characterize and monitor patient-specific genomic alterations in AML, in order to tailor treatments and allow early detection of expanding subclonal population. Moreover, future studies are needed to define the hypomethylation pattern resulting from the described genomic lesions and its cooperation with differentiation stage-specific histone modification in the regulation of the leukemogenic transcriptional program.

\section{Data Availability}

The clinical and molecular data used to support the findings of this study are included within the article.

\section{Conflicts of Interest}

Giovanni Martinelli receives compensation as a consultant for ARIAD/INCYTE, Pfizer, Celgene, Amgen, J\&J, and Roche. The other authors declare no conflicts of interest.

\section{Authors' Contributions}

Samantha Bruno and Maria Teresa Bochicchio equally contributed.

\section{Acknowledgments}

This work was supported by the European Union's Seventh Framework Programme (FP7/2007-2013) (under grant agreement no. 306242-NGS-PTL) and by the Associazione Italiana per la Ricerca sul Cancro (AIRC) (Investigator Grant no. 19226 to Giovanni Martinelli).

\section{References}

[1] T. Cancer and G. Atlas, "Genomic and epigenomic landscapes of adult de novo acute myeloid leukemia the cancer genome atlas research network," New England Journal of Medicine, vol. 368, no. 22, pp. 2059-2074, 2013.

[2] M. A. McDevitt, "Clinical applications of epigenetic markers and epigenetic profiling in myeloid malignancies," Seminars in Oncology, vol. 39, no. 1, pp. 109-122, 2012.

[3] S. F. Chaudry and T. J. T. Chevassut, "Epigenetic guardian: a review of the DNA methyltransferase DNMT3A in acute myeloid leukaemia and clonal haematopoiesis," BioMed Research International, vol. 2017, Article ID 5473197, 13 pages, 2017.

[4] S. J. Chen, Y. Shen, and Z. Chen, "A panoramic view of acute myeloid leukemia," Nature Genetics, vol. 45, no. 6, pp. 586-587, 2013.

[5] R. Rampal, A. Alkalin, J. Madzo et al., "DNA hydroxymethylation profiling reveals that WT1 mutations result in loss of TET2 function in acute myeloid leukemia," Cell Reports, vol. 9, no. 5, pp. 1841-1855, 2014.

[6] E. Pronier, R. L. Bowman, J. Ahn et al., "Genetic and epigenetic evolution as a contributor to WT1-mutant leukemogenesis," Blood, vol. 132, no. 12, pp. 1265-1278, 2018.

[7] T. J. Ley, L. Ding, M. J. Walter et al., "DNMT3A mutations in acute myeloid leukemia," New England Journal of Medicine, vol. 363, no. 25, pp. 2424-2433, 2010.

[8] L. I. Shlush, S. Zandi, A. Mitchell et al., "Identification of preleukaemic haematopoietic stem cells in acute leukaemia," Nature, vol. 506, no. 7488, pp. 328-333, 2014.

[9] H. P. Koeffler and G. Leong, "Preleukemia: one name, many meanings," Leukemia, vol. 31, no. 3, pp. 534-542, 2017.

[10] G. Genovese, A. K. Kähler, R. E. Handsaker et al., "Clonal hematopoiesis and blood-cancer risk inferred from blood DNA sequence," New England Journal of Medicine, vol. 371, no. 26, pp. 2487-2477, 2014. 
[11] S. Jaiswal, P. Fontanillas, J. Flannick et al., "Age-related clonal hematopoiesis associated with adverse outcomes," New England Journal of Medicine, vol. 371, no. 26, pp. 2488-2498, 2014.

[12] C. B. Cole, D. A. Russler-Germain, S. Ketkar et al., "Haploinsufficiency for DNA methyltransferase $3 \mathrm{~A}$ predisposes hematopoietic cells to myeloid malignancies," Journal of Clinical Investigation, vol. 127, no. 10, pp. 3657-3674, 2017.

[13] X. J. Yan, J. Xu, Z. H. Gu et al., "Exome sequencing identifies somatic mutations of DNA methyltransferase gene DNMT3A in acute monocytic leukemia," Nature Genetics, vol. 43, no. 4, pp. 309-315, 2011.

[14] G. Marcucci, K. H. Metzeler, S. Schwind et al., "Age-related prognostic impact of different types of DNMT3A mutations in adults with primary cytogenetically normal acute myeloid leukemia," Journal of Clinical Oncology, vol. 30, no. 7, pp. 742-750, 2012.

[15] A. F. T. Ribeiro, M. Pratcorona, C. Erpelinck-Verschueren et al., "Mutant DNMT3A: a marker of poor prognosis in acute myeloid leukemia," Blood, vol. 119, no. 24, pp. 5824-5831, 2012.

[16] A. Renneville, N. Boissel, O. Nibourel et al., "Prognostic significance of DNA methyltransferase $3 \mathrm{~A}$ mutations in cytogenetically normal acute myeloid leukemia: a study by the acute leukemia french association," Leukemia, vol. 26, no. 6, pp. 1247-1254, 2012.

[17] F. Thol, F. Damm, A. Lüdeking et al., "Incidence and prognostic influence of DNMT3A mutations in acute myeloid leukemia," Journal of Clinical Oncology, vol. 29, no. 21, pp. 2889-2896, 2011.

[18] H. A. Hou, Y. Y. Kuo, C. Y. Liu et al., "DNMT3A mutations in acute myeloid leukemia: stability during disease evolution and clinical implications," Blood, vol. 119, no. 2, pp. 559-568, 2012.

[19] K. Morita, H. M. Kantarjian, F. Wang et al., "Clearance of somatic mutations at remission and the risk of relapse in acute myeloid leukemia," Journal of Clinical Oncology, vol. 36, no. 18, pp. 1788-1797, 2018.

[20] B. Bhatnagar, A. K. Eisfeld, D. Nicolet et al., "Persistence of DNMT3A R882 mutations during remission does not adversely affect outcomes of patients with acute myeloid leukaemia," British Journal of Haematology, vol. 175, no. 2, pp. 226-236, 2016.

[21] C. Gebhard, D. Glatz, L. Schwarzfischer et al., "Profiling of aberrant DNA methylation in acute myeloid leukemia reveals subclasses of CG-rich regions with epigenetic or genetic association," Leukemia, vol. 33, no. 1, pp. 26-36, 2019.

[22] M. Wang, S. Zhang, S.-S. Chuang et al., "Angioimmunoblastic $\mathrm{T}$ cell lymphoma: novel molecular insights by mutation profiling," Oncotarget, vol. 8, no. 11, 2017.

[23] I. Fried, C. Bodner, M. M. Pichler et al., "Frequency, onset and clinical impact of somatic DNMT3A mutations in therapyrelated and secondary acute myeloid leukemia," Haematologica, vol. 97, no. 2, pp. 246-250, 2012.

[24] H. DöhnerDöhner, E. Estey, D. Grimwade et al., "Diagnosis and management of AML in adults: 2017 ELN recommendations from an international expert panel," Blood, vol. 129, no. 4, pp. 424-447, 2017.

[25] E. Papaemmanuil, M. Gerstung, L. Bullinger et al., "Genomic classification and prognosis in acute myeloid leukemia," New England Journal of Medicine, vol. 374, no. 23, pp. 2209-2221, 2016.

[26] J. P. J. Issa, G. Roboz, D. Rizzieri et al., "Safety and tolerability of guadecitabine (SGI-110) in patients with myelodysplastic syndrome and acute myeloid leukaemia: a multicentre, randomised, dose-escalation phase 1 study," Lancet Oncology, vol. 16, no. 9, pp. 1099-1110, 2015.

[27] W. Chung, A. D. Kelly, P. Kropf et al., "Genomic and epigenomic predictors of response to guadecitabine in relapsed/ refractory acute myelogenous leukemia," Clinical Epigenetics, vol. 11, no. 1, 2019.

[28] R. Itzykson, O. Kosmider, T. Cluzeau et al., "Impact of TET2 mutations on response rate to azacitidine in myelodysplastic syndromes and low blast count acute myeloid leukemias," Leukemia, vol. 25, no. 7, pp. 1147-1152, 2011.

[29] R. Bejar, A. Lord, K. Stevenson et al., "TET2 mutations predict response to hypomethylating agents in myelodysplastic syndrome patients," Blood, vol. 124, no. 17, pp. 2705-2712, 2014.

[30] D. Russo, M. Malagola, A. De Vivo et al., "Multicentre phase III trial on fludarabine, cytarabine (ara-C), and idarubicin versus idarubicin, ara- $\mathrm{C}$ and etoposide for induction treatment of younger, newly diagnosed acute myeloid leukaemia patients," British Journal of Haematology, vol. 131, no. 2, pp. 172-179, 2005.

[31] D. Russo, G. Pricolo, M. Michieli et al., "Fludarabine, arabinosyl cytosine and idarubicin (FLAI) for remission induction in poor-risk acute myeloid leukemia," Leukemia \& Lymphoma, vol. 40, no. 3-4, pp. 335-343, 2001.

[32] M. Rothenberg-Thurley, S. Amler, D. Goerlich et al., "Persistence of pre-leukemic clones during first remission and risk of relapse in acute myeloid leukemia," Leukemia, vol. 32, no. 7, pp. 1598-1608, 2018.

[33] D. H. Spencer, D. A. Russler-Germain, S. Ketkar et al., "CpG island hypermethylation mediated by DNMT3A is a consequence of AML progression," Cell, vol. 168, no. 5, pp. 801-816.e13, 2017.

[34] A. D. Kelly, J. Madzo, P. Madireddi et al., "Demethylator phenotypes in acute myeloid leukemia," Leukemia, vol. 32, no. 10, pp. 2178-2188, 2018.

[35] Y. Qu, L. Siggens, L. Cordeddu et al., "Cancer-specific changes in DNA methylation reveal aberrant silencing and activation of enhancers in leukemia," Blood, vol. 129, no. 7, pp. e13-e25, 2017.

[36] P. Hirsch, Y. Zhang, R. Tang et al., "Genetic hierarchy and temporal variegation in the clonal history of acute myeloid leukaemia," Nature Communications, vol. 7, no. 1, 2016.

[37] C. C. Smith, A. Paguirigan, G. R. Jeschke et al., "Heterogeneous resistance to quizartinib in acute myeloid leukemia revealed by single-cell analysis," Blood, vol. 130, no. 1, pp. 48-58, 2017.

[38] N. Potter, F. Miraki-Moud, L. Ermini et al., "Single cell analysis of clonal architecture in acute myeloid leukaemia," Leukemia, vol. 33, no. 5, pp. 1113-1123, 2019.

[39] C. M. McMahon, T. Ferng, J. Canaani et al., "Clonal selection with RAS pathway activation mediates secondary clinical resistance to selective FLT3 inhibition in acute myeloid leukemia," Cancer Discovery, vol. 9, no. 8, pp. 1050-1063, 2019. 


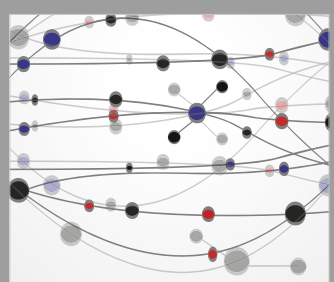

The Scientific World Journal
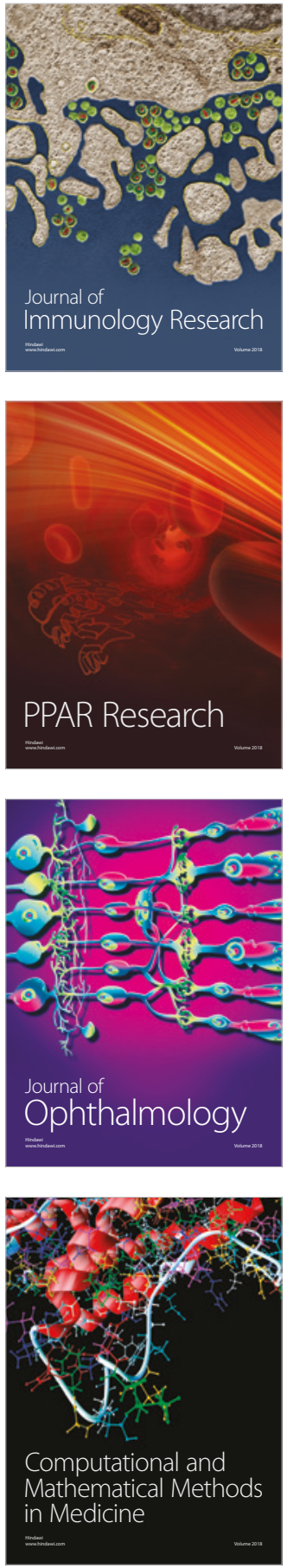

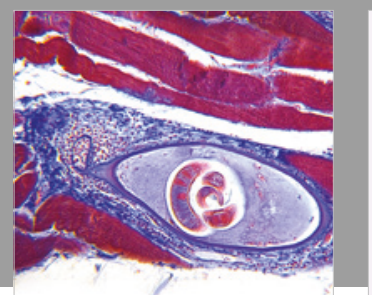

Gastroenterology Research and Practice

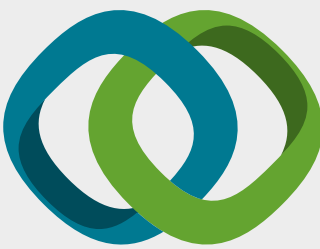

\section{Hindawi}

Submit your manuscripts at

www.hindawi.com
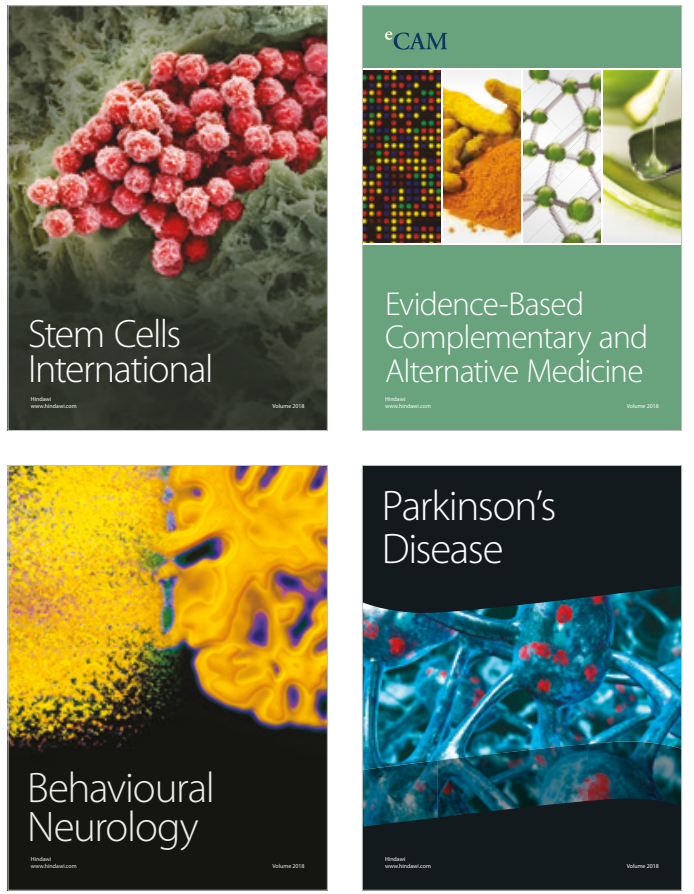

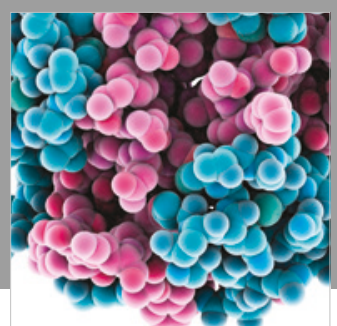

ournal of

Diabetes Research

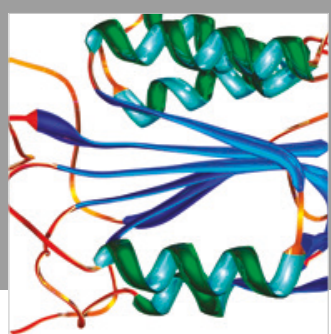

Disease Markers
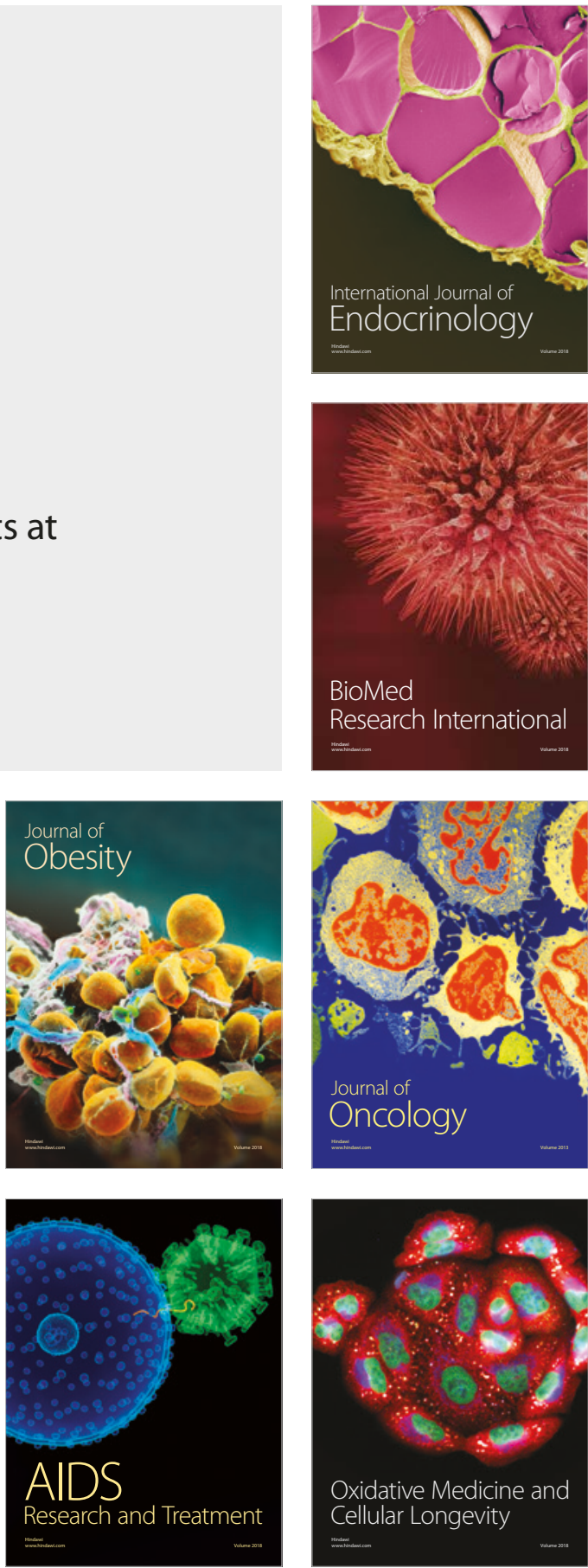